\title{
Sparse Reconstruction of Multi-component Doppler Signature Exploiting Target Dynamics
}

\author{
Saurav Subedi ${ }^{\dagger}$, Yimin D. Zhang ${ }^{\ddagger}$, and Moeness G. Amin ${ }^{\dagger}$ \\ $\dagger$ Center for Advanced Communications, Villanova University, Villanova, PA 19085, USA \\ $\ddagger$ Department of Electrical and Computer Engineering, Temple University, Philadelphia, PA 19122, USA
}

\begin{abstract}
In this paper, we propose a novel technique for a non-parametric sparse reconstruction of the Doppler signature of multiple ground moving targets exploiting prior knowledge about the target dynamics. The Doppler signature of multiple nonstationary targets is commonly modeled as a multi-component polynomial phase signal, whose order depends on the complexity of target dynamics and the coherent processing interval. In recent years, sparse signal reconstruction and compressive sensing algorithms have been extensively deployed in both parametric and non-parametric estimation of Doppler signatures. The performance of sparsity based Doppler signature reconstruction algorithms is significantly compromised in the presence of strong noise and a large number of missing samples. Herein, we present an effective technique for a non-parametric sparse reconstruction of a multi-component Doppler signature by exploiting the a priori information on target dynamics. Simulation results show that the proposed technique significantly outperforms the conventional sparse reconstruction based methods in such challenging conditions.
\end{abstract}

\section{INTRODUCTION}

The Doppler signature of multiple non-stationary targets is commonly modeled as a multi-component general order polynomial phase signal $[1,2]$, whose order depends on the complexity of target dynamics and the coherent processing interval. A reliable estimation of the Doppler signature, characterizing the target motion, is an important problem in many radar applications, including localization, motion parameter estimation and tracking (e.g., [3-5]).

Time-frequency (TF) representations are widely used for analyzing non-stationary Doppler signatures arising from a complex target trajectory. Spectrograms are the most fundamental TF representations, which perform the short-time Fourier transform (STFT) over small overlapping/non-overlapping segments of temporal samples obtained via the application of a window (e.g., rectangular, Hamming, and Hanning). Spectrograms generally suffer from limited spectral resolution and smearing in the TF representations as a result of windowing in the time-domain. Several other advanced TF representation methods have been developed over the years to deal with different types of signals. For example, the WignerVille distribution (WVD) exhibits excellent performance for TF analysis and estimation of instantaneous frequency (IF) of single-component linear frequency modulated (LFM) signals. However, it introduces cross-terms in the analysis of multicomponent signals, resulting in a significant performance degradation [6]. Cohen's class of TF representations, which reduces the artifacts due to cross-terms by low-pass filtering the signal in the ambiguity domain, have been widely used in the analysis of multi-component linear and non-linear frequency modulated (FM) signals [7, 8]. The adaptive optimal kernel (AOK) is a commonly used data-dependent kernel that generally seek auto-term preservation and cross-term suppression [9]. Complex-time distribution (CTD) is another class of TF representations introduced to highly concentrate phase derivatives in the $\mathrm{TF}$ plane that provide accurate IF tracking for signals with fast varying IF [10].

In many practical applications, the observation suffers from a high proportion of missing samples, due to fading, shadowing or removal of impulse noise. Missing samples introduce noise-like artifacts spread all over the TF plane. As such, it becomes difficult to estimate the Doppler signature, accurately and reliably, using traditional TF representations. In recent years, sparse reconstruction and compressive sensing algorithms have been successfully applied for reconstruction of TF signatures [11-13]. To mitigate the effects of missing samples, several approaches, such as application of signal adaptive kernels $[11,14,15]$ and exploitation of the continuous structure of the TF signature as a prior [16], have been proposed for effective reconstruction of TF signatures. A generalized approach to exploit sparse reconstruction techniques to obtain quadratic TF distributions based on optimal multi-task kernel design and from compressed measurements observed in the time domain or the joint-variable domain is presented in [17]. However, these methods do not exploit the prior knowledge about the target dynamics, which, as we show in this paper, can be effectively utilized to mitigate the effects of high percentage of missing samples and strong noise at a low computational cost.

In this paper, we consider a problem of estimating the Doppler signature corresponding to multi-target trajectories at a Doppler sensor operating in a passive bistatic radar (PBR) configuration. The measurement vector comprises a high percentage of missing samples and is corrupted by a strong additive noise. In such situations, the performance of the conventional sparse-reconstruction based method degrades significantly. We exploit the prior knowledge of the target dynamics and the multi-target state estimates of the preceding observation interval to pre-estimate the Doppler frequencies and provide a supplementary layer of intelligence to the sparse reconstruction algorithm through a feedback mechanism. Simulation results are provided to validate the ability of the proposed technique to provide an improved performance over the conventional method. 
Notations: A lower (upper) case bold letter denotes a vector (matrix). Specifically, $\mathbf{I}_{N}$ and $\mathbf{0}_{N}$ denote the $N \times N$ identity and zero matrices, respectively. (.)* (. $)^{T}$, and $(.)^{H}$, respectively, denote complex conjugation, transpose, and hermitian operations, and $\circ$ denotes the Hadamard product. $\mathbb{R}^{n \times 1}$ and $\mathbb{C}^{n \times 1}$, respectively, represent the $n$-dimensional real and complex vectors. $\|\cdot\|_{1}$ and $\|\cdot\|$, respectively, denote the $l_{1}$ and $l_{2}$ norm of a vector, whereas $\Re($.$) and \Im($.$) , respectively, stand$ for the real and imaginary parts of a complex number, and $x \sim \mathcal{N}(a, b)$ denotes variable $x$ to be a Gaussian distributed with mean $a$ and variance $b$. In addition, $\operatorname{diag}($.$) and \operatorname{tr}($.$) ,$ respectively, denote the diagonal and trace operations.

\section{SIGNAL MODEL}

We consider $N$ ground moving targets under surveillance in a PBR system. The PBR configuration comprises a broadcast station transmitting at a known carrier frequency $f_{c}$ and a single Doppler sensor. The transmitter is assumed to be located at $\mathbf{b}$, whereas the sensor is located at $\mathbf{r}$. The transmitter and the receiver are assumed stationary and their locations are precisely known a priori.

The problem considered in this paper is to accurately reconstruct the Doppler signature corresponding to the motion of $N$ targets by processing the signal received at the Doppler sensor over a period of $T$ seconds. Considering the sampling frequency of $F_{s} \mathrm{~Hz}$, the total number of samples over the entire observation period is equal to $N_{s}=F_{s} T$. It is assumed that, over the entire observation period of $T$ seconds, the Doppler sensor generates $K$ Doppler frequency estimates, at a periodic interval $\Delta$, denoted as $k=1, \cdots, K$. As such, for the estimation of instantaneous Doppler frequency over each interval, the sensor exploits $L=N_{s} / K$ time samples.

The state vector of the $n$th target at the $k$ th observation, $\mathbf{x}_{k, n}$, represents a point in the state space and comprises its instantaneous position $\mathbf{p}_{k, n} \triangleq\left[p_{x, k, n}, p_{y, k, n}\right]^{T}$ and velocity $\mathbf{v}_{k, n} \triangleq\left[v_{x, k, n}, v_{y, k, n}\right]^{T}$ in the two-dimensional (2-D) Cartesian coordinate system, i.e.,

$$
\mathbf{x}_{k, n}=\left[\mathbf{p}_{k, n}^{T}, \mathbf{v}_{k, n}^{T}\right]^{T} .
$$

As such, the multi-target state set at the $k$ th observation is given as $\mathcal{X}_{k}=\left\{\mathbf{x}_{k, 1}, \cdots, \mathbf{x}_{k, N}\right\}$.

The target dynamics is modeled as a linear Gaussian nearlyconstant velocity model, such that

$$
\mathbf{x}_{k, n}=\mathbf{F} \mathbf{x}_{k-1, n}+\mathbf{G w}_{k, n},
$$

where $\mathbf{F}$ is the state transition matrix defined as

$$
\mathbf{F}=\left[\begin{array}{cc}
\mathbf{I}_{2} & \Delta \mathbf{I}_{2} \\
\mathbf{0}_{2} & \mathbf{I}_{2}
\end{array}\right],
$$

and $\mathbf{w}_{k, i} \sim \mathcal{N}\left(\mathbf{0}, \sigma_{w}^{2} \mathbf{I}_{2}\right)$ is the process noise modeled as additive white Gaussian random variable. The transition matrix

$$
\mathbf{G}=\left[\begin{array}{c}
\frac{\Delta^{2}}{2} \mathbf{I}_{2} \\
\Delta \mathbf{I}_{2}
\end{array}\right]
$$

accounts for the small acceleration that could deviate the target trajectory from being strictly linear. It is assumed that the target motion is rectilinear and uniform within an observation interval. As such, the process noise covariance is defined as

$$
\mathbf{Q}=\sigma_{\mathbf{w}}^{2}\left[\begin{array}{cc}
\frac{\Delta^{4}}{4} \mathbf{I}_{2} & \frac{\Delta^{3}}{2} \mathbf{I}_{2} \\
\frac{\Delta^{3}}{2} \mathbf{I}_{2} & \Delta^{2} \mathbf{I}_{2}
\end{array}\right],
$$

where $\sigma_{\mathbf{w}}^{2}$ is the variance of the process noise.

The $l$ th sample of the $k$ th interval is a superposition of signals reflected from $N$ targets, such that,

$$
y_{k}(l)=\sum_{n=1}^{N} \alpha_{k, n} \exp \left(-\jmath 2 \pi f_{k, n} l\right)+e_{k}(l),
$$

where $\alpha_{k, n}$ is the target reflection coefficient corresponding to the $n$th target, $l=0, \cdots, L-1$ represent the discrete time instants over the $k$ th interval, $\jmath=\sqrt{-1}, e_{k}(l) \sim \mathcal{N}\left(0, \sigma_{e}^{2}\right)$ is the additive noise, and $f_{k, n}$ is the bistatic Doppler frequency given as [4]

$$
f_{k, n}=-\frac{\mathbf{v}_{k, n}^{T}}{\lambda}\left[\frac{\mathbf{p}_{k, n}-\mathbf{r}}{\left\|\mathbf{p}_{k, n}-\mathbf{r}\right\|}+\frac{\mathbf{p}_{k, n}-\mathbf{b}}{\left\|\mathbf{p}_{k, n}-\mathbf{b}\right\|}\right],
$$

where $\lambda=c / f_{c}$ is the wavelength of the transmitted signal, $c$ is the velocity of propagation of a radio signal in free space. The Doppler measurement space or the field-of-view is defined over an interval $\left[-f_{0},+f_{0}\right][3]$, where $f_{0}$ denotes the maximum possible Doppler shift. As such, for the $k$ th interval we obtain the $\mathbb{C}^{L \times 1}$ vector $\tilde{\mathbf{y}}_{k}=\left[y_{k}(1), \cdots, y_{k}(L)\right]^{T}$.

As discussed in Section I, in many applications the observation suffers a high proportion of missing samples, due to fading, shadowing or impulse noise, which introduces noise-like artifacts in the TF plane, rendering it difficult to estimate the Doppler signature, accurately and reliably, using traditional TF representations. In such situations, sparse signal reconstruction and compressive sensing algorithms have been commonly deployed. Let us consider $M$ samples missing from the $\mathbb{C}^{L \times 1}$ vector $\tilde{\mathbf{y}}_{k}$, where $0 \leq M<L$. The missing sample positions are assumed to be randomly and uniformly distributed over time. Accounting for the missing samples, we define an observation vector $\mathbf{y}_{k}$, such that

$$
\mathbf{y}_{k}=\tilde{\mathbf{y}}_{k} \circ \mathbf{m}_{k},
$$

where

$$
\mathbf{m}_{k}=\left\{\begin{array}{cc}
1, & \text { if } l_{k} \in \mathcal{S}_{k}, \\
0, & \text { otherwise }
\end{array}\right.
$$

with $\mathcal{S}_{k} \subset\{1, \cdots, L\}$ denoting the set of observed time instants and its cardinality is $\left|\mathcal{S}_{k}\right|=L-M$.

The emphasis is on the exploitation of the prior knowledge about the target dynamics through a feedback mechanism for a reliable and accurate estimation of the Doppler signature. As such, we resort to the basic procedure for sparse reconstruction directly exploiting the time samples as the observation vectors. Herein, we compare the performance of the sparse reconstruction method, with and without such feedback. It is noted, however, that a superior performance may be achieved by exploiting the Fourier relationship between the TF domain and the instantaneous auto-correlation function (IAF) domain, together with the application of AOK for mitigating the effects of missing samples in the TF plane [16]. 


\section{DOPPLER FREQUENCY ESTIMATION USING}

CONVENTIONAL SPARSE SIGNAL RECONSTRUCTION

For exploiting sparse signal reconstruction, we base our formulation on the Fourier relationship between the observation vector $\mathbf{y}_{k}$ and its spectral components. The sparse vector $\mathbf{u}_{k}$ is to be estimated, which contains all the TF representation entries for the $k$ th observation interval. As such, we can define a linear relationship

$$
\mathbf{y}_{k}=\boldsymbol{\Phi} \mathbf{u}_{k}+\mathbf{e}_{k}
$$

where $\boldsymbol{\Phi}$ represents an oversampled IDFT matrix, i.e., $L \ll$ $N_{f}$ and $\mathbf{e}_{k}=\left[e_{k}(1), \cdots, e_{k}(L)\right]^{T}$ represents the additive noise Gaussian vector. As such, the entire Doppler measurement space over the interval $\left[-f_{0},+f_{0}\right]$ is represented by $N_{f}$ discrete frequencies. The problem of (10) can be formulated as the following $l_{1}$-norm minimization problem,

$$
\min \left\|\mathbf{u}_{k}\right\|_{1} \quad \text { subject to }\left\|\mathbf{y}_{k}-\boldsymbol{\Phi} \mathbf{u}_{k}\right\|^{2} \leq \epsilon
$$

where $\epsilon$ is the pre-defined error level. The minimization problem in (11) can be readily solved using a number of methods available for sparse signal reconstruction.

The performance of conventional sparse Doppler signature reconstruction algorithms is significantly compromised in the presence of strong noise and large number of missing samples. In the following, we present an effective technique for a non-parametric sparse reconstruction of a multi-component Doppler signature by exploiting the a priori information on target dynamics.

\section{Proposed METHOD}

The target dynamics is known a priori as defined in (2), and we assume that the estimated target state from the previous observation instant $\hat{\mathcal{X}}_{k-1}=\left\{\hat{\mathbf{x}}_{k-1,1}, \cdots, \hat{\mathbf{x}}_{k-1, N}\right\}$ is available at the sensor. ${ }^{1}$ As such, the target state for the next observation can be pre-estimated as $\overline{\mathcal{X}}_{k}=\left\{\overline{\mathbf{x}}_{k, 1}, \cdots, \overline{\mathbf{x}}_{k, N}\right\}$, where

$$
\overline{\mathbf{x}}_{k, n}=\mathbf{F} \hat{\mathbf{x}}_{k-1, n},
$$

with $\overline{\mathbf{x}}_{k, n}=\left[\overline{\mathbf{p}}_{k, n}^{T}, \overline{\mathbf{v}}_{k, n}^{T}\right]^{T}$. Accordingly, we can pre-estimate the set of Doppler frequency measurements for the $k$ th observation instant, $\overline{\mathcal{F}}_{k}=\left\{\bar{f}_{k, 1}, \cdots, \bar{f}_{k, N}\right\}$, where

$$
\bar{f}_{k, n}=-\frac{\overline{\mathbf{v}}_{k, n}^{T}}{\lambda}\left[\frac{\overline{\mathbf{p}}_{k, n}-\mathbf{r}}{\left\|\overline{\mathbf{p}}_{k, n}-\mathbf{r}\right\|}+\frac{\overline{\mathbf{p}}_{k, n}-\mathbf{b}}{\left\|\overline{\mathbf{p}}_{k, n}-\mathbf{b}\right\|}\right] \text {. }
$$

Following (6), we can define samples of pre-estimated observation vector for the $k$ th interval as

$$
\bar{y}_{k}(l)=\sum_{n=1}^{N} \exp \left(-\jmath 2 \pi \bar{f}_{k, n} l\right)
$$

resulting in a $\mathbb{C}^{L \times 1}$ vector $\overline{\mathbf{y}}_{k}=\left[\bar{y}_{k}(1), \cdots, \bar{y}_{k}(L)\right]^{T}$. In order to exploit the prior knowledge of target dynamics, we redefine the measurement vector as a weighted sum of the current observation vector $\mathbf{y}_{k}$ and the pre-estimated observation vector

\footnotetext{
${ }^{1}$ In practice, a multi-sensor multi-target tracking algorithm is deployed to obtain such pre-estimates, as described in [5]. The impact of exploiting the method proposed herein in the overall multi-sensor multi-target tracking problem will be discussed in the future work.
}

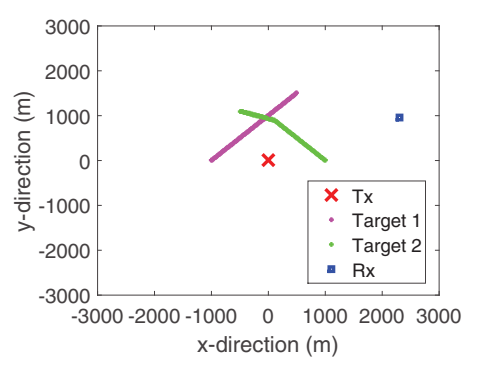

Fig. 1. Passive bistatic radar tracking the movement of multiple targets.

$\overline{\mathbf{y}}_{k}$, such that the sparse reconstruction problem in (10) is reformulated as

$$
\beta \mathbf{y}_{k}+(1-\beta) \overline{\mathbf{y}}_{k}=\mathbf{\Phi} \mathbf{z}_{k}+\mathbf{e}_{k},
$$

where $\mathbf{z}_{k}$ represents the unknown sparse vector, $\beta$ determines the relative weights assigned to the current observation and the pre-estimated observation vector. The higher the proportion of missing samples in the current observation, the higher weight is assigned to the pre-estimated observation vector. It is noted that a smaller $\beta$ puts a lower emphasis on the current observation, which mitigates the effect of missing sample and strong additive noise. On the other hand, it results in a slower adaptation to any sudden changes in target trajectories. The solution to the underlying sparse reconstruction problem in (15) is obtained by solving the following $l_{1}$-norm minimization problem,

$\min \left\|\mathbf{z}_{k}\right\|_{1} \quad$ subject to $\left\|\left(\beta \mathbf{y}_{k}+(1-\beta) \overline{\mathbf{y}}_{k}\right)-\mathbf{\Phi} \mathbf{z}_{k}\right\|^{2} \leq \epsilon$.

The solution of the $l_{1}$-norm minimization technique practically converges to an $N$-sparse solution through multi-modal optimization that minimizes the mean absolute error. In this paper, we use orthogonal matching pursuit (OMP) [18] for the sparse reconstruction because the number of targets are assumed to be known a priori. As such, the $k$ th observation instant, we obtain estimates of instantaneous Doppler frequencies corresponding to the $N$ ground moving targets,

$$
\hat{\mathcal{F}}_{k}=\left\{\hat{f}_{k, 1}, \cdots, \hat{f}_{k, N}\right\} .
$$

In the following, we present simulation results to demonstrate that the performance can be significantly improved by exploiting prior information on target dynamics, particularly when the observation signal is subject to strong noise and high percentage of missing samples.

\section{Simulation Results}

In the simulations, we consider a PBR configuration as illustrated in Fig. 1, where a broadcast station is located at the origin and transmitting at $950 \mathrm{MHz}$, and the Doppler frequency measurement sensor is located at a distance of $2.5 \mathrm{~km}$ from the transmitter. The region of surveillance is assumed to be a rectangular area bounded by $[-2500,2500]^{T} \mathrm{~m}$ along both the $x$ - and $y$-axes. This example represents a scenario with two targets with intersecting trajectories and one of the targets changes its velocity at some instant during the observation period. We consider two targets initially located at $[-1000,0]^{T}$ 


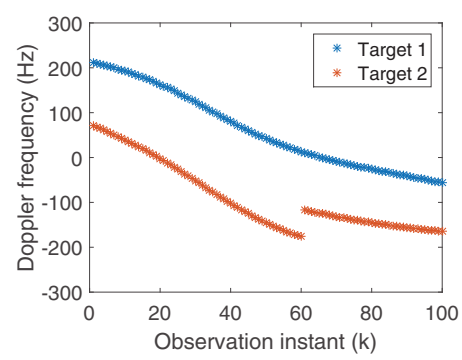

Fig. 2. True Doppler signature.

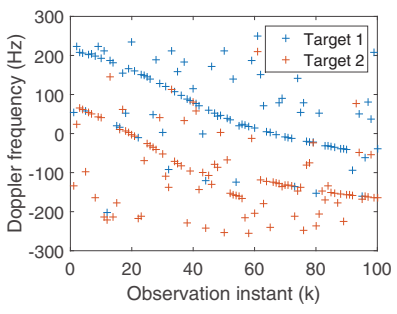

(a)

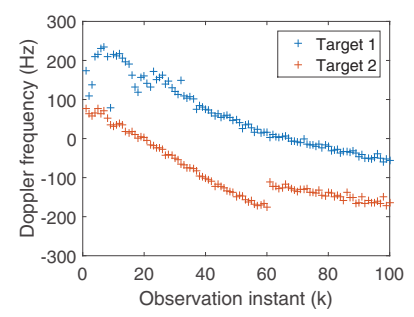

(b)
Fig. 3. Doppler signature reconstruction (a) Conventional method. (b) Proposed method.

$\mathrm{m}$ and $[1000,0]^{T} \mathrm{~m}$ and travelling along linear trajectories with velocities $[30,30]^{T} \mathrm{~m} / \mathrm{s}$ and $[-30,30]^{T} \mathrm{~m} / \mathrm{s}$, respectively, as shown in Fig. 1. The second target changes its trajectory and follows a velocity of $[-30,10]^{T} \mathrm{~m} / \mathrm{s}$ at $k=61$.

We consider an overall observation period of $T=50$ seconds divided into $K=100$ equal intervals each of period $\Delta=0.5 \mathrm{~s}$, such that each interval consists of 256 time samples obtained at a sampling rate of $512 \mathrm{~Hz}$. The ground truth of the Doppler signature observed at the Doppler sensor monitoring the surveillance area over the entire observation period is shown in Fig. 2. In order to demonstrate the effect of high proportion of missing samples and strong noise, we consider $75 \%$ samples missing in each interval, and the SNR is defined to be $-10 \mathrm{~dB}$. In such a situation, the performance of the conventional sparse reconstruction method is not reliable as shown in Fig. 3(a). The estimated Doppler signature is discontinuous and scattered, respectively, due to several false estimates and missed detections.

On the other hand, as shown in Fig. 3(b), the proposed method benefits from the exploitation of the prior knowledge of the target dynamics and the target state estimates from the previous observation interval. In this simulation, we use $\beta=0.5$ to assign equal relative weights to the current observation vector and the predicted observation vector. The performance improvement over the conventional method depends on the accuracy of target state estimates obtained from the previous observation interval. This is evident in Fig. 3(b), as the accuracy of Doppler estimates improves with time, which in turn, contributes to a better estimation of target state estimates through a closed-loop process.

\section{CONCLUSIONS}

In this paper, we have proposed a scheme for reliably estimating the Doppler signature at a Doppler sensor operating in a passive bistatic radar configuration, where the received signal is assumed to be corrupted by strong additive noise and a high proportion of missing samples. The proposed method exploits the prior knowledge about the target dynamics and provides an additional layer of information in the form of pre-estimated observation vector to the sparse reconstruction algorithm through a feedback mechanism. Simulation results verified a significant performance improvement over the conventional sparse reconstruction methods that do not utilize the knowledge of target dynamics.

\section{REFERENCES}

[1] S. Peleg and B. Porat, "The Cramer-Rao lower bound for signals with constant amplitude and polynomial phase," IEEE Trans. Signal Process., vol. 39, no. 3, pp. 749-752, Mar. 1991.

[2] S. Saha and S. M. Kay, "Maximum likelihood parameter estimation of superimposed chiprs using monte carlo importance sampling," IEEE Trans. Signal Process., vol. 50, no. 2, pp. 224-230, Feb. 2002.

[3] B. Ristic and A. Farina, "Target tracking via multi-static Doppler shifts," IET Radar, Sonar and Navig., vol. 7, no. 5, pp. 508-516, Jun. 2013.

[4] Y. D. Zhang and B. Himed, "Moving target parameter estimation and SFN ghost rejection in multistatic passive radar," in Proc. IEEE Radar Conf., Ottawa, Canada, Apr. 2013, pp. 1-5.

[5] S. Subedi, Y. D. Zhang, M. G. Amin, and B. Himed, "Group sparsity based multi-target tracking in multi-static passive radar systems using Doppler-only measurements," in Proc. IEEE Intl. Radar Conf., Arlington, VA, May 2015, pp. 880-885.

[6] S. Barbarossa, "Analysis of multicomponent LFM signals by a combined Wigner-Hough transform," IEEE Trans. Signal Process., vol. 43, no. 6, pp. 1511-1515, Jun. 1995.

[7] H. I. Choi and W. J. Williams, "Improved time-frequency representation of multicomponent signals using exponential kernels," IEEE Trans. Acoust. Speech and Signal Process., vol. 37, no. 6, pp. 862-871, Jun. 1989.

[8] J. Jechang and W. J. Williams, "Kernel design for reduced interference distributions," IEEE Trans. Signal Process., vol. 40, no. 2, pp. 402-412, Feb. 1992.

[9] D. L. Jones and R. G. Baraniuk, "An adaptive optimal-kernel timefrequency representation," IEEE Trans. Signal Process., vol. 43, no. 10, pp. 2361-2371, Oct. 1995.

[10] S. Stanković and L. J. Stanković, "Introducing time-frequency distribution with a 'complex-time' argument," Electr. Lett., vol. 32, no. 14, pp. 1265-1267, Jul. 1996.

[11] Y. D. Zhang, M. G. Amin, and B. Himed, "Reduced interference timefrequency representations and sparse reconstruction of undersampled data," in Proc. EUSIPCO, Marrakech, Morocco, Sep. 2013, pp. 1-5.

[12] M. G. Amin, Y. D. Zhang, and B. Jokanovic, "Time-frequency signature reconstruction from random observations using multiple measurement vectors," in Proc. ICASSP, Florence, Italy, May 2014, pp. 345-349.

[13] L. Stankovic, S. Stankovic, I. Orovic, and Y. D. Zhang, "Timefrequency analysis of micro-Doppler signals based on compressive sensing," in M. G. Amin (ed.), Compressive Sensing for Urban Radars, CRC Press, 2014.

[14] Y. D. Zhang and M. G. Amin, "Compressive sensing in nonstationary array processing using bilinear transforms," in Proc. IEEE SAM Signal Proc. Workshop, Hoboken, NJ, Jun. 2012, pp. 349-352.

[15] B. Jokanovic, M. G. Amin, Y. D. Zhang, and F. Ahmad, "Timefrequency kernel design for sparse joint-variable signal representations," in Proc. EUSIPCO, Lisbon, Portugal, Sep. 2014, pp. 2100-2104.

[16] Q. Wu, Y. D. Zhang, and M. G. Amin, "Continuous structure based Bayesian compressive sensing for sparse reconstruction of timefrequency distributions," in Proc. Intl. Conf. DSP, Hong Kong, China, Aug. 2014, pp. 831-836.

[17] M. Amin, B. Jokanovic, Y. Zhang, and F. Ahmad, "A sparsity perspective to quadratic time-frequency distributions," Digital Signal Process. (in press).

[18] J. A. Tropp and A. C. Gilbert, "Signal recovery from random measurements via orthogonal matching pursuit," IEEE Trans. Info. Theory, vol. 53, no. 12 , pp. 4655-4666, Dec. 2007. 